\title{
The disordered boundary of the cell: emerging properties of membrane-bound intrinsically disordered proteins
}

https://doi.org/10.1515/bmc-2019-0003

received November 27, 2018; accepted January 28, 2019.

\begin{abstract}
We define the disordered boundary of the cell (DBC) as the system formed by membrane tethered intrinsically disordered protein regions, dynamically coupled to the underlying membrane.

The emerging properties of the DBC makes it a global system of study, which cannot be understood from the individual properties of their components. Similarly, the properties of lipid bilayers cannot be understood from just the sum of the properties of individual lipid molecules.
\end{abstract}

The highly anisotropic confined environment, restricting the position and orientation of interacting sites, is affecting the properties of individual disordered proteins. In fact, the collective effect caused by high concentrations of disordered proteins extend beyond the sum of individual effects.

Examples of emerging properties of the DBC include enhanced protein-protein interactions, protein-driven phase separations, Z-compartmentalization, and protein modulated electrostatics.

Keywords: Intrinsically disordered proteins; Membrane proteins; Cell Boundaries; Sgnalling environment; Bio nano composites; Crowding; Liquid phase separation; Z-compartmentalization; Src family kinases.

*Corresponding author: Miquel Pons, BioNMR Laboratory, Inorganic and Organic Chemistry Department, University of Barcelona, Baldiri Reixac 10-12, 08028 Barcelona, Spain, E-mail:mpons@ub.edu Irrem-Laareb Mohammad: BioNMR Laboratory, Inorganic and Organic Chemistry Department, University of Barcelona, Baldiri Reixac 10-12, 08028 Barcelona, Spain

Borja Mateos: Max F. Perutz Laboratories, Department of Computational and Structural Biology, University of Vienna, Campus Vienna Biocenter 5, 1030 Vienna, Austria

\section{Introduction}

Compartmentalization is an essential feature of cells and life that allows spatiotemporal control of processes. Lipid membranes define the boundary of the cell and cellular compartments. Within the cells, compartments may also be generated in phase-separated regions, like the lipid driven raft-like structures in membranes, or in the liquid protein or protein/RNA droplets. Information is transmitted across compartments through dynamic protein interaction networks, which acquire, amplify, transmit, and eventually respond to signals that are channelled along the signaling cascades.

Regarding the protein interaction networks, standard paradigms assume that: (i) the functionally relevant regions of proteins are folded domains, (ii) protein complexes adopt well-defined 3D structures, and (iii) interaction networks obey an isotropic and unrestricted reaction-diffusion model. Each of these paradigms has been challenged by recent findings. First, a large proportion of eukaryotic proteins, ranging from one [1] to two thirds [2], depending on the criteria used, are predicted to be intrinsically disordered proteins (IDPs). Interestingly, IDPs are far less abundant in prokaryotic organisms, suggesting that some emerging properties associated with extensive protein flexibility may have enabled the jump in complexity from bacteria and archaea to multi-cellular organisms [3]. Secondly, some complexes formed by two or more IDPs remain disordered [4] and some IDPs participate in liquidlike phases that demix from homogeneous solutions [5]. Also, a network of IDP interactions contributes to the selectivity of nuclear pores [6] or cell-to-cell channels [7]. Finally, essential signaling interactions communicating the cell with its environment take place in the highly anisotropic vicinity of lipid membranes [8].

The region extending into the cytoplasm from membrane-associated proteins is especially rich in intrinsically disordered regions (IDR). Iakoucheva et al. reported that $70 \%$ of transmembrane proteins involved 
in signaling have IDRs longer than 30 residues [9]. The enrichment of IDRs in the proximity of lipid membranes might be just the consequence of the association of IDRs with the needed signaling at the cell interface. We suggest that there is a boundary layer enriched with disordered domains coupled to the lipid bilayer that provides a singular environment, with properties distinct from the bulk cytoplasm or an isolated membrane model. We name this region, which includes the lipid membrane and its boundary layer, as the disordered boundary of the cell (DBC). We refer to the IDP-rich region of the cytoplasm in direct contact with the lipid bilayer, as the $\varepsilon$-layer (Figure 1). In this perspective article, we want to discuss the emergent properties expected for the $\varepsilon$-layer and their possible implications in cell signaling. While disordered regions appear more frequently in the cytoplasmic side of the membrane, some of the concepts are equally applicable to the other side of the membrane, forming the boundary with the extracellular matrix.

\section{Cell membrane and boundary regions}

Membrane models emphasize the lipid-dominated hydrophobic environment with embedded proteins. Models have been updated regularly since the original "fluid mosaic" concept of Singer and Nicholson [10]. Engelman emphasized the crowded behaviour and heterogeneity of membrane protein composition [11]. Successive studies shed light on the structural complexity and continuous dynamics undergoing in cellular boundaries as lipid phases, lipid-protein interactions, macromolecular polymer adsorption, membrane curvature, and flip-flop lipid motions, among others nicely reviewed by Goñi et al. [12].

Membrane attached proteins, cytoplasmic regions of transmembrane proteins, or cytoplasmic proteins adsorbed on the membrane surface play important roles in coupling the membrane to the bulk cytoplasm. The interactions of the cytoplasmic regions of membrane embedded proteins and the underlying actin cytoskeleton may restrict lipid diffusion and create membrane compartments [13]. Abundant long coiled-coil proteins, called golgins, attached to the golgi have been suggested to create a tentacular matrix helping to recruit Rab-coated vesicles [14]. The emphasis of this review is, however, on the expected emergent properties when the membrane associated protein chains are disordered and present at high local concentrations.
The aqueous layer in contact with the hydrophobic membrane is a boundary layer. In purely physical terms, the potential of boundary layers to generate emergent properties is well-known. However, the $\varepsilon$-layer is predicted to have unique features that arise from its "composite" nature. Composites are materials combining different constituents with very distinct individual properties, that when mixed together, render a material with novel properties [15]. Well-known examples in other fields are reinforced concrete or animal bones, in which metal bars or collagen fibers modify a homogeneous matrix of cement or apatite, respectively. In contrast to these macroscopic examples, the $\varepsilon$-layer is not a rigid material, but has the properties of a fluid. The IDPs act as polymers anchored to the fluid lipid membrane that fill-in and modulate the properties in its immediate vicinity. The matrix would be the surrounding solution experiencing strong electrostatic and hydrodynamic fields.

In an isolated lipid membrane, the electric and hydrodynamic fields would show smooth continuous distributions, with gradients originating from the proximity of the charged bounding layer. However, the presence of abundant charged residues, a characteristic feature of the IDP sequences, introduce discontinuities in the $\varepsilon$-layer. The position of these charges is determined by their interaction with the electric field generated on the lipid surface or originate from neighboring charged residues. Additionally, the location of the perturbing charges is modulated by constraints on the IDP network.

Considering that IDPs have nanoscopic dimensions and are dynamically coupled to their environment, the DBC can be described as a fluid nano-composite which emphasizes the essential roles of its small scale and complex dynamics.

\section{Abundance of intrinsically disordered proteins in membranes}

The abundance of IDRs is a hallmark of eukaryotes and is functionally associated to their higher regulatory complexity as compared to prokaryotic organisms. IDR are more abundant in the subset of transmembrane human proteins than in the complete proteome $(57.4 \%$ versus $35.2 \%$ defined as the percentage of proteins having consecutive regions longer than 30 residues predicted to be disordered by DISOPRED2 [16, 17]). This implies that about $\sim 45 \%$ of the IDPs are transmembrane proteins. The cytoplasmic side of membrane proteins is particularly enriched in IDRs [18]. 


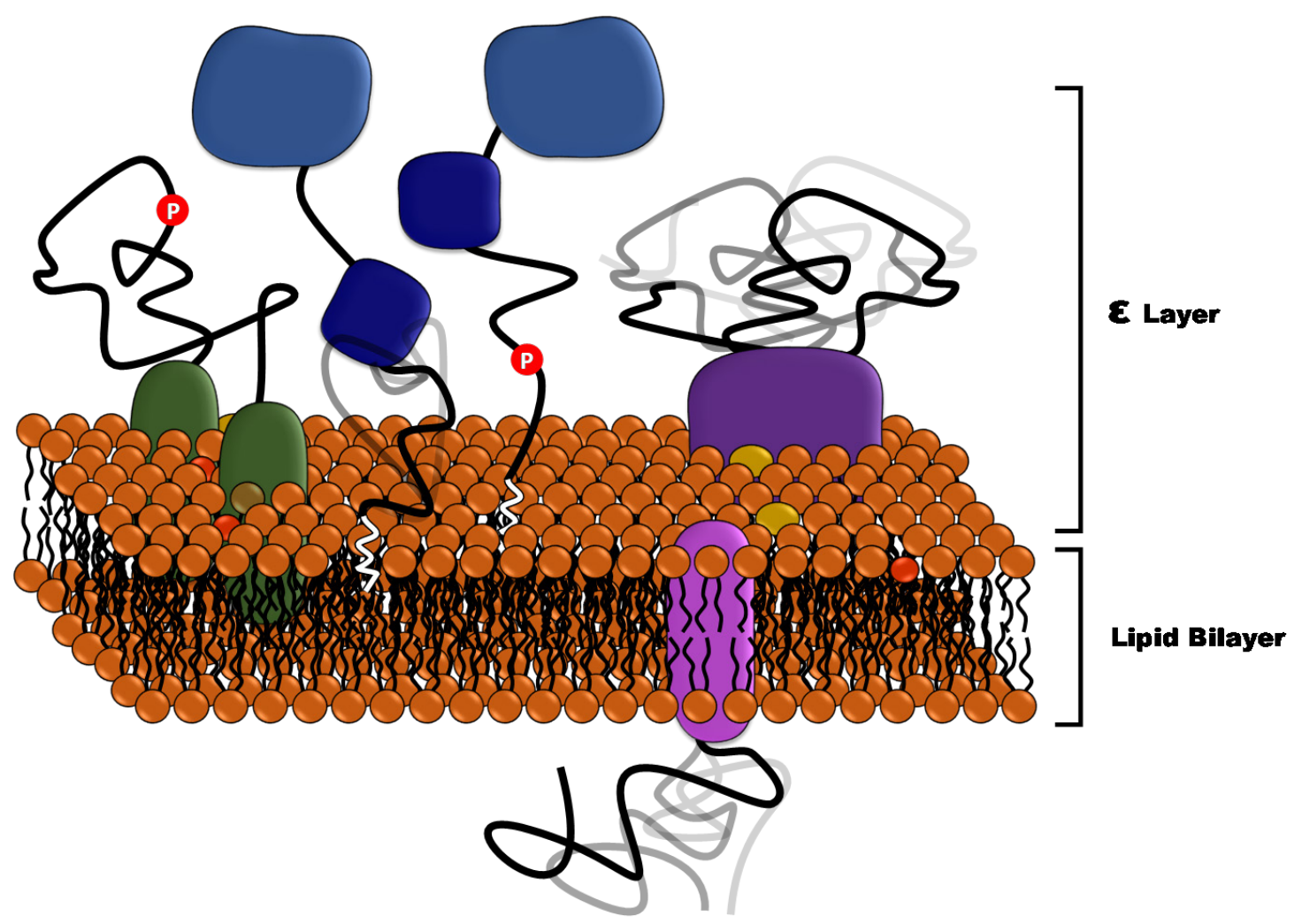

\section{The Disordered Boundary of the Cell}

Figure 1: The disordered boundary of the cell. The e-layer is defined as the region occupied by the water soluble IDPs tethered to the membrane surface. Due to the high local concentration of the IDPs, the properties of the e-layer are not just the sum of the features of the individual proteins, in the same way as the properties of the lipid bilayer are not the sum of the features of individual lipids. The coupling between the e-layer and the underlying membrane in the DBC is reminiscent of the coupling between the polar headgroups and the hydrophobic lipid interior. Analogous to the distinct environment provided by the lipids to integral membrane proteins, the $\varepsilon$-layer also provides a singular environment for folded domains that are immersed in it, although the nature of the interactions is indeed very different in the hydrophobic core of the membrane and the hydrophilic e-layer.

Non-integral cytoplasmic proteins anchored to the membrane by long intrinsically disordered regions terminated by covalently bound lipid chains, such as a myristoyl or a palmitoyl groups, represent an additional source of IDPs in the $\varepsilon$-layer. Prominent examples are MARCKS (myristoylated alanine rich C kinase substrate), the alpha subunits of $\mathrm{G}$ proteins [19], and the members of the Src family of kinases (SFKs) [20]. The combined abundance per cell of these IDP families is 15\% of that of actin, the major component of the cytoskeleton [21].

The abundance of IDPs that interact in a permanent or reversible fashion with the membrane surface, unveils a scenario with a high density of disordered proteins confined in a narrow perimembranal layer. Such high density would result in emergent properties, i.e. beyond the simple juxtaposition of the individual components. The critical concentration at which emerging properties would appear will depend on the nature of the actual
IDPs and the underlying lipid composition. For instance, simulations on the phase separation and gelation of multivalent proteins with disordered linkers emphasize as relevant determinants: the fraction of charged residues in the linkers that affects the effective solvation volume, the average spatial distance between residues, and the cooperativity of the interactions between individual interacting regions [22].

Less abundant but still highly relevant, are those proteins with exposed disordered segments in the extracellular part of lipid bilayer, specially enriched in endocytic processes [23] and cell-matrix or cell-cell interactions [24]. Although there is a small number of proteins with long extracellular disordered regions (more than 200 residues), these are usually highly glycosylated cell adhesion molecules, also called proteoglycans. The CD44 antigen is a prototypical example, containing a variable disordered stem region that expands from 80 to 


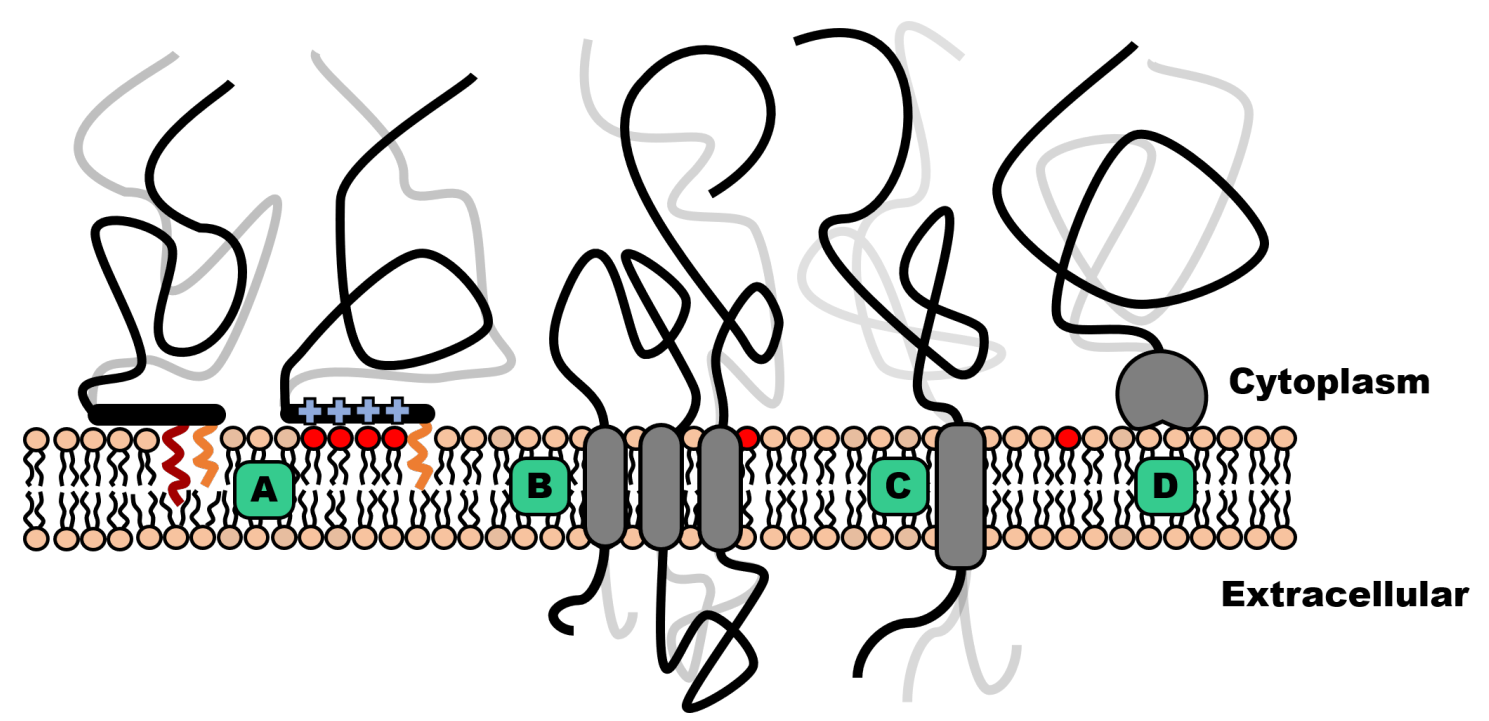

Figure 2: Anchoring of IDRs to the membrane surface. A. Lipid anchors. B. Polytopic membrane proteins. C. Single pass integral proteins. D. Attachment based on globular lipid binding domains.

almost 500 residues depending on alternative splicing $[25$, 26]. The cytoplasmic and extracellular loops connecting the transmembrane helices of integral polytopic proteins are often disordered and play crucial roles in substrate recognition and receptor activation/repression (Figure $2 \mathrm{~B}$ and $2 \mathrm{C}$ ). The extracellular loops of G-protein coupled receptors (GPCRs) are paradigmatic examples. They have an average length of 50 residues and have key functions for ligand recognition $[25,27]$.

Some of the general concepts related to IDP dynamics in the proximity of membranes are equally applicable to both sides of the membrane, albeit major differences exist between them. The interior of the cell presents an overall larger density in IDRs, with special highlight on the role of IDRs from non-transmembrane proteins that undergo reversible binding to the membranes (Figure $2 \mathrm{~A}$ and 2D). Also, the cytoplasmic side accumulates negatively charged lipids. The emphasis of this manuscript is on the cytoplasmic disordered boundary of the cell.

\section{Tethering disordered proteins to the membrane through lipid anchors}

Lipid anchors allow the reversible association of proteins to the cytoplasmic side of the cell membrane [28]. Examples of lipid anchors include the N-terminal myristoylation that occurs in all SFKs and in the $\alpha$-subunit of heterotrimeric G-proteins. Additional palmitoylation of cysteine regions is observed in most SFKs and $\mathrm{G} \alpha$-proteins
(Figure 2A). Palmitoylation also occur in the cytoplasmic disordered tail of GPCRs constitutively connected to the integral membrane region. Thus, in this case, the lipid anchor probably acts as a modulator and is not the main driver of the protein-lipid interactions [29].

Small monomeric GTPases of the Ras family are modified at their C-terminus by isoprenoid moieties: farnesyl (3 isoprene units, 15 carbons) or geranylgeranyl (4 isoprene units, 20 carbons) located at the end of a Hypervariable Region. Additional palmitoylation of a second cysteine occurs in most Ras proteins [19]. The two-signal hypothesis indicates that two-lipid binding moieties are required for stable attachment of peripheral membrane proteins [28]. In the case of c-Src and K-Ras, that have only one lipid modification, N-terminal myristoylation and C-terminal farnesylation, respectively, a polybasic sequence seems to provide the required additional phospholipid-binding element (Figure 2A).

The myristoylated alanine rich c-kinase substrate (MARCKS) [30] is an abundant $35 \mathrm{kDa}$ intrinsically disordered protein that contains a N-terminal myristoylation site and an effector domain in the center of the protein. This domain contains multiple positively charged lysine residues that provide an electrostatic attachment to negatively charged lipids. Additionally, it has multiple serine residues that, when phosphorylated by C-kinase or Rho kinase, can prevent the electrostatic interaction causing the protein detachment from the membrane into the cytosol. This is known as an electrostatic switch [31]. 


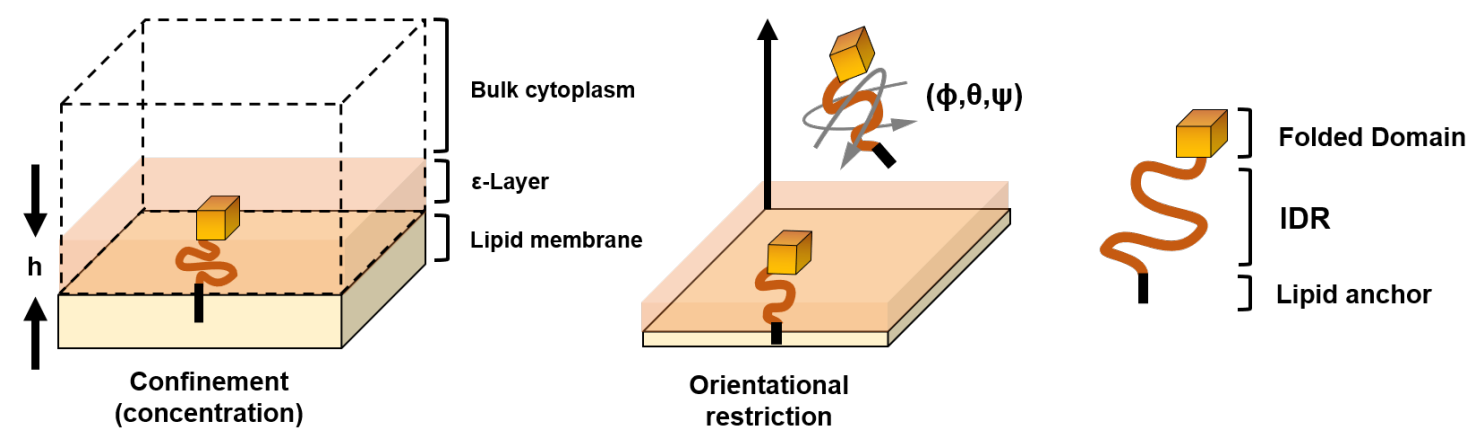

Figure 3: Scaling from 3D to 2D. Confinement to two dimensions reduces one of the dimensions to an effective "confinement length of dimensions comparable to those of the attached IDP (left). Additional interactions (steric or electrostatic) restrict the orientation of elements capable of interactions reducing the "productive" collisions. On the other hand, the preexisting conformational restrictions decrease the entropic penalty of intermolecular complexes between confined molecules.

A reciprocal effect of MARCKS on the lipid distribution has also been reported, affecting the distribution of PI(4,5) $\mathrm{P}_{2}$, which is sequestered by the unphosphorylated effector domain, probably forming a neutral complex bringing together two or three $\mathrm{PI}(4,5) \mathrm{P}_{2}$ in a tight complex with the protein [32]. Thus, MARCKS provides an illustrative example of the possible cross-talk between the lipid and IDP systems in the DBC.

\section{Emerging properties of the DBC}

The emergent properties of the IDP-populated $\varepsilon$-boundary are a potential determinant of the function of the cell frontier. The disordered character of the anchored proteins has important implications at various levels: i) the modulation of the intrinsic properties of the individual IDR arising from the fact that they are tethered on the membrane surface; ii) the implications on the specific interactions between the membrane attached IDR and other signaling partners located in the proximity of the membrane, being themselves intrinsically disordered or globular; and iii) the modulation of the environment of the cell boundary affecting other components, which may include components not physically attached to the cell membrane but located in its proximity.

At the level of individual membrane anchored IDR, their conformational dynamics will be modulated by steric effects and by the presence of strong electrostatic fields. These fields are created by the negatively charged cytoplasmic membrane surface and by the abundant charged residues present in most IDRs.

The properties of individual IDR are distinctly manifested in their direct interactions. The interaction space available in the vicinity of the membrane is much more restricted than in an isotropic 3D scenario. To this extent, if a specific binding site is attached to a membrane anchored IDR, how would its potential interactions be modified?

\section{Dimensional scaling and rotational restriction}

Scaling of the dissociation constants from 3D to 2D has two well defined components, illustrated in Figure 3: First the collapse from an isotropic volume to a confined layer of width $h$ results in an effective change in the concentration terms. The work of Bell et al. describes a model in which the dissociation constants between 3D and 2D would simply be scaled by an effective confinement length so that $\mathrm{K}_{d}{ }^{2 \mathrm{D}}=$ $h \cdot \mathrm{K}_{\mathrm{d}}{ }^{3 \mathrm{D}}$ [33]. Secondly a restriction in the orientation of the interacting elements, so that the probability of productive encounters between potential partners resulting from their approaching in the proper orientation, is reduced [34].

Interestingly, as shown in Honig's simulations [34], the conformational restriction induced by membrane anchoring may actually favor the clustering of membraneattached proteins. The entropy loss intrinsic to any complex formation can be lower, if the potential interacting partners show a reduced conformational landscape due to their membrane attachment.

In Honig's approach, membrane attachment plays only a restrictive role in the possible orientations. It was explicitly assumed that the energetic contributions from binding event would be identical in the isotropic 3D scenario and in the confined environment of the membrane. If we imagine that binding arises from the cooperative interaction between multiple weakly binding 


\section{Multisite interaction in register}

Orientational bias

B IDR modulated IDR crowding
(entropic)
D Z-compartimentalization (selection)
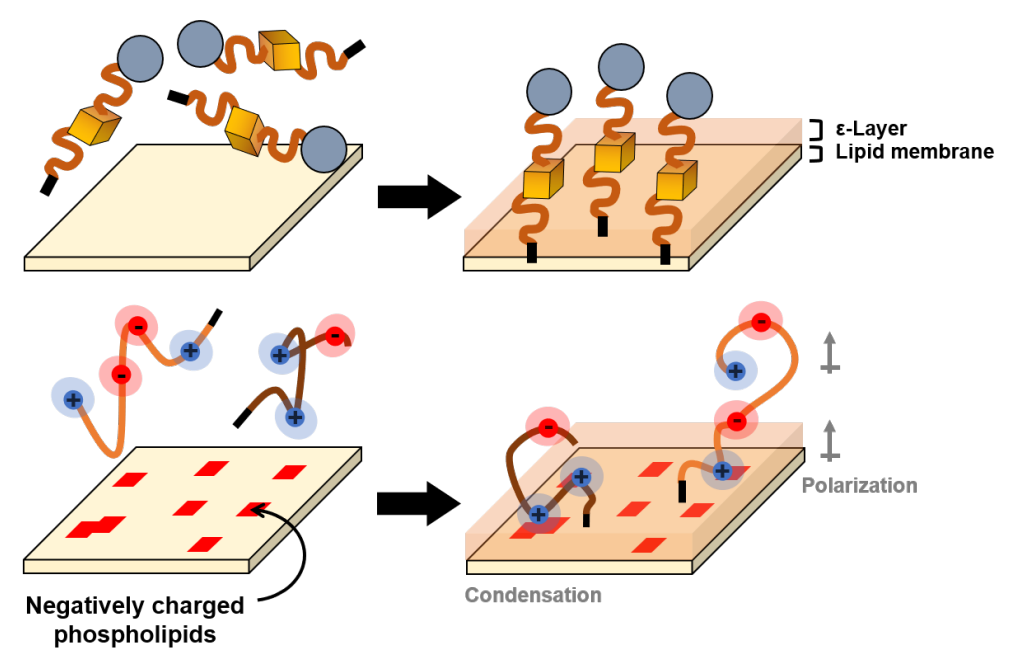

phospholipids
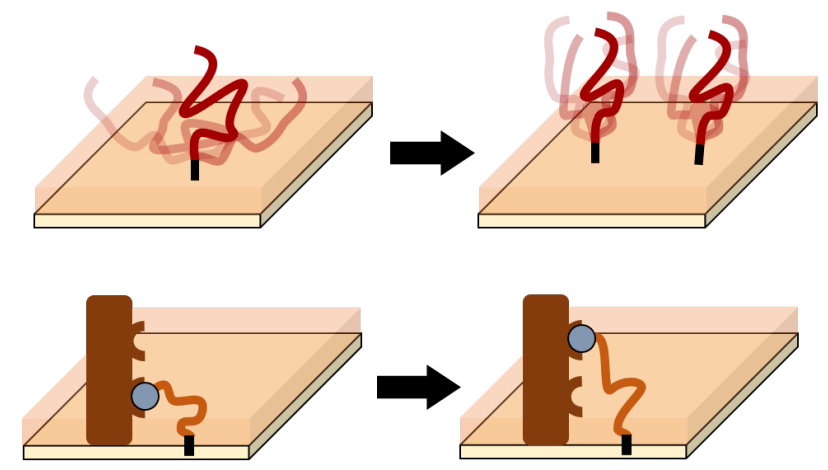

Figure 4: Emerging properties of the DBC. A. Enhancement of lateral interactions by placing interacting sites "on register" through membrane anchoring. B. Electrostatic interactions with the charged lipid membrane may affect the positional distribution of charged residues modulating the protein conformational space and the extension of the electrostatic effects propagated through dipolar interactions generated from oppositely charged residues in the IDP. C. IDP crowding has an entropic origin, resulting from the mutual restriction of the conformational space sampled by approaching membrane tethered IDPs. D. Z-compartmentalization refers to the selection of interacting partners/substrates depending on their location with respect to the membrane surface.

sites, not necessarily located contiguously along the IDR sequence, the anisotropic environment in the cell boundary can enforce distinct relative distributions of the individual sites with respect to the membrane surface.

This distribution can be strongly affected, for example, by the relative placement of charged residues and residues forming interaction sites along the membrane-anchored IDR sequence. Membrane anchoring can enhance lateral interactions by ensuring that multiple interacting sites are in register, as illustrated in Figure 4A.

Because of their density in the DBC, IDR may cause environmental effects that modulate the chemical potential of other chemical species, even if they are not physically attached to the membrane.

At least two effects are expected to be important: i) IDR induced changes in the effective polarizability of the boundary and ii) IDR induced crowding.

\section{IDR modulated electrostatics}

The electrostatic field originating on the negatively charged cytoplasmic lipid surface determines the equilibrium distribution of diffusing charged species in the boundary. While individual cations and anions can diffuse independently to reach equilibrium distributions, the positional distribution of charged residues in an IDR is defined by their protein sequence and depends on their conformational landscape. Thus, the electrostatic potential field is modulated by the presence of membrane attached IDRs. In parallel, the polarization of the IDR dipoles may actually extend the electrostatic influence of the charged membrane to longer distances than would be expected in an IDR-free membrane boundary (Figure 4B). 


\section{IDR crowding}

IDR crowding (Figure 4C) represents a distinct situation from the well-known excluded volume crowding effects predicted for globular proteins. Specifically that, the centers of two rigid spherical objects cannot approach closer than the sum of their radii. Thus, the effective volume that can be occupied by one of the objects is reduced by the volume taken by the total number of the other objects present in the solution [35]. Two main IDP features depart from the simplest non-interacting hardspheres model of crowding. First, their dynamic nature introduces a strong entropic component, and, secondly their highly accessible surface that facilitates non-specific interactions with other components of the cytoplasm, sometimes referred to as quinary interactions [36].

In the case of IDPs, the volume occupied by the molecule in a given instant is much smaller than the total volume sampled by the same molecule over a long time. At a same density of anchored proteins, IDRs can cover a larger surface than globular proteins [37]. When two IDPs approach, although they could occupy the same region of space, they cannot do it simultaneously. Therefore, their conformational space becomes restricted. The result is an entropic penalty that hinders the approximation of two IDR below a certain distance.

Crowding of membrane attached IDP is also modulated by the excluded volume arising from the proximity of the membrane. Convex membrane curvature around the protein attachment site increases the accessible volume that can be sampled by the IDP and therefore alleviates the entropic penalty.

Membrane bending by anchored polymers were theoretically predicted in 1995 [38]. Experimentally, the work of J. Stachowiak et al. has recently demonstrated the important role that disordered segments play in curvature formation and shape sensing. One of the most well-studies protein domains responsible for curvature sensing are BAR domains, that contain amphipathic helices and long disordered tails. At a fixed surface density, determined by the interaction of the helical segments, convex curvature of the surface reduces the entropic penalty associated with the interaction of the IDR. Modulation of the local rigidity in disordered regions is a sophisticated mechanism used by BAR proteins for sensing curvature [39]. Conversely, enforcing the approximation of membrane anchored IDPs, for example by the interaction of neighbor globular domains, can induce membrane curvature [40].

The environmental effects of the IDR-rich DBC will affect all processes taking place in the $\varepsilon$-layer, including globular domains or other molecules not physically attached to the membrane. Thus, modifications of membrane attached IDRs, e.g. post translational modifications, may affect processes that are not, apparently, directly related to the modified IDP.

\section{Z-compartmentalization}

Compartmentalization is often found as a regulatory strategy restricting the possible partners for a given interaction by their common distribution in mutually excluding sites. In the cell boundary, the localization of interaction sites along the direction perpendicular to the membrane surface, the Z-direction, may generate an alternative compartmentalization mechanism (Figure 4D).

A example of Z-compartmentalization is illustrated by the ROCK2 kinase, a Rho-associated coiled coil kinase formed by constitutively active kinase dimers separated from the membrane anchoring regulatory domain by a long $(107 \mathrm{~nm})$ coiled-coil. The length of the coiled-coil is well conserved, although the sequence itself is variable, and consistent with its functioning as a molecular ruler. Truncation of the coiled-coil does not affect the kinase activity in vitro, but resulted in a complete loss of actin stress fibers when expressed in vivo. It has been suggested that ROCK2 activity is ultimately determined by the proximity of the substrate to the kinase domain, which would link phosphorylation to the distance to the membrane surface [41, 42]. MARCKS protein is a ROCK2 substrate [43].

While the rigid coiled coil provides a well-defined ruler, disordered linkers in the boundary of the cell may offer a modulation mechanism enabling or restricting the accessibility of the active site to specific substrates by changes in the flexibility of the connecting region, e.g. through post-translational modifications or alternative splicing changing the protein effective length [44]. A recent example shows that the length of the hypervariable domain (HVD) of Rab GTPases connecting their nucleotide binding domain (NBD) from their membrane anchoring, hydrophobic prenyl group, determine the differences in selectivity of two Rabs interacting with the same catalytic site. The Rabs are located at different distances from the membrane surface, resulting in spatial selectivity. A short HVD prevents the NBD from reaching the active site that stabilizes an otherwise reversible interaction with the membrane, but the effect can be reversed by artificially extending the length of the HVD [45]. 


\section{Protein phase separation}

Historically, "nucleolar accessory bodies", later called Cajal bodies, were already described by Santiago Ramon y Cajal in 1903 in neuronal cells. These granules lack a surrounding phospholipid membrane and have remained obscure for a long time. One century later, it was demonstrated that these supramolecular assemblies have liquid properties, and are generated by phase separation of proteins and/or RNA. Recently, more examples have been described in biology and phase separation has emerged as a rediscovered field, but now involving cell biology, supramolecular chemistry, and polymer physics $[5,46]$. Phase separation is a demixing transition driven by preferential interactions or selective exclusion within a homogeneous solution. Multiple weak interactions become cooperative when the interaction sites are part of a polymer but retain a highly dynamic exchange, resulting in liquid-like properties. The proteins associated with liquid-phases are mainly IDPs or low-complexity regions present in disordered segments. Phase transitions in multivalent signaling proteins have been suggested to be important in signaling [47].

Most of these spatially organized regions have liquid properties and are observed as "droplets" due to the surface tension. However, it is important to note that liquid-liquid phases generated by proteins and/or RNA deviate conceptually from the well-known van der Waals fluid, comprising spherical particles with isotropic interactions. Macromolecular polymers like proteins are highly anisotropic and protein-driven separation of protein fluid phases may be enhanced in the $\varepsilon$-layer.

Existing examples are the nephrin and LAT receptors. Nephrin receptor binds to Nck and the neural WiskottAldrich syndrome protein (N-WASP) and form phaseseparated liquid droplets in vitro [47]. In presence of lipid bilayers and in cells, at much lower concentrations, this assembly is reflect in form of membrane clusters [48]. Similarly, the interaction between the linker for activation of T cells (LAT), the growth factor receptor-bound protein 2 (GRB2), the GRB2-related adaptor protein 2 (GADS), the son of sevenless (SOS), and the SH2 domain-containing leukocyte of $76 \mathrm{kDa}$ (SLP76) undergoes phase separation in vitro and generates membrane puncta in cells upon $\mathrm{T}$ cell receptor [49].

At least two $\varepsilon$-layer-specific features may enhance selfdriven clustering of membrane-bound IDPs, positional matching of interaction sites and orientation bias. These two can be understood as an enhanced concentration of the interaction sites with respect to an isotropic solution. Positional matching implies a concentration of the interacting species by transferring from 3D to 2D. Orientation bias reflects the reduced entropic penalty associated to the cooperative interaction between two pairs of sites in separate molecules when these molecules adopt predominantly parallel dispositions, e.g. because both are tethered to the membrane surface.

\section{The disordered region of Src family kinases: fuzzy complexes and membrane induced dimerization.}

Recent studies of the membrane tethered, non-receptor SFKs have uncovered the importance of their intrinsically disordered domains and provide interesting examples of emerging properties associated to the DBC. The nine members of the SFK family have common domain architectures, with three folded domains and an $\mathrm{N}$-terminal IDR that includes the membrane anchoring SH4 domain and the Unique domain [20]. The name "Unique" refers to the lack of homology of this region among the various SFKs that contrasts with the high conservation of the globular domains, including the kinase domain (SH1) and two classical regulatory domains ( $\mathrm{SH} 3$ and $\mathrm{SH} 2$ ). Myristoylation of the SH4 domain and membrane anchoring are required for c-Src activity [50]. Titration with lipid bicelles monitored by NMR have identified additional lipid binding regions, in the Unique and $\mathrm{SH} 3$ domains [51]. The N-terminal myristoyl moiety binds to the $\mathrm{SH} 3$ domain in the absence of lipids, but is inserted into the lipid bilayer in the presence of lipid membrane (e.g. large unilamelar vesicles (LUV)). The SH3 domain nucleates an intramolecular fuzzy complex in which the Unique and SH4 domains are loosely tethered around the globular domain, while retaining their disordered character [52]. The intramolecular fuzzy complex is retained in the membrane-bound form bringing the $\mathrm{SH} 3$ domain in close proximity to the lipid surface [53]. Thus, globular domains "coated" with disordered proteins can also be an integral part of the DBC (Figure 5).

Interestingly, mutations that deactivate the lipidbinding region in the Unique domain result in a 50\% decrease in the invasive capacity of Src-dependent colorectal cancer cells [52]. However, recent results show that in a LUV-anchored form of c-Src, containing the myristoylated SH4, Unique, and SH3 domains, the additional lipid binding site in the Unique domain is not interacting directly with the liposome. This lipid interacting region, contributes to stabilize the intramolecular interaction of the myristoyl group with the 

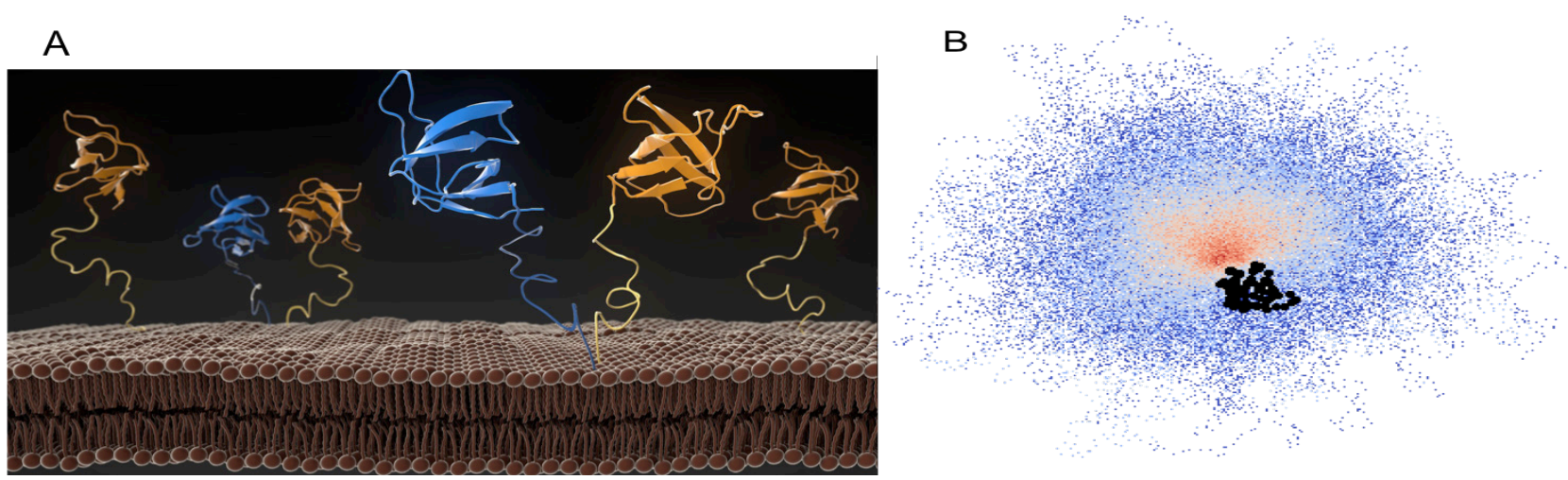

Figure 5: c-Src N-terminal region. A. Artist view of the dimerization of c-Src by its myristoylated SH4 domain when anchored to lipid membranes (adapted from Chem. Select cover figure to reference 56 under CC-BY-NC license). B. Relative volumes of the SH3 domain (black) and the space visited by adjacent disordered domains, represented by the cloud formed by the position of the Ca carbons in an ensemble compatible with SAXS experimental data.

SH3 domain in the non-membrane bound form [53] and modulates the orientation of the globular domains with respect to the membrane surface, via the intramolecular fuzzy complex. The change in orientation may explain the observed change in substrate specificity caused by mutations in the Unique lipid-binding region [Roche, S. private communication, manuscript in preparation], although more complex scenarios cannot be ruled out.

A recent report has suggested that the myristoyl group could also interact with the kinase domain of a second Src molecule to form a dimer [54]. The intermolecular interaction of the Src kinase domain with a N-terminal myristoyl group is expected to be promiscuous. Thus, the number of possible heterodimers is very large and interactions involving the kinase domain may affect its activity as well as the substrate preference, in addition to being modulated by the competing insertion of the myristoyl group in the lipid bilayer.

Dimerization on the surface of supported lipid bilayers had been previously observed for the N-terminal region of Src without the kinase domain $[55,56]$. In contrast to the transient binding observed by monomeric c-Src molecules to supported lipid bilayers, the membrane bound dimers were persistently attached and could be detected by antibodies even after extensive washing. Persistent binding is consistent with the simultaneous insertion of two myristoyl groups by c-Src dimers. Dimerization was not observed in solution or when the protein was deposited on a poly-lysine coated surface, thus the presence of phospholipids seems to be a requirement for membrane induced dimerization. Persistent binding was also observed in pure zwitterionic phosphatidyl choline supported bilayers, ruling out the possible partition of Src into phase-separated lipid regions.
The positive charges arising from the initial lysine rich segment (K5, K7, and K9) followed by a stretch of three arginine residues (R14, R15, and R16) contribute to increase the binding to negatively charged lipids but, surprisingly, also enhance the formation of dimers, in spite of the expected electrostatic repulsion

Membrane induced oligomerization was observed in constructs containing the myristoylated SH4 domain attached to fluorescent proteins [57,58]. Extensive oligomerization was observed for short myristoylated peptides containing the entire SH4 region (15 residues) [55] or even in a myristoylated (2-9) c-Src peptide [59]. Interestingly, the first ten $\mathrm{SH} 4$ residues have a distinct role in the stabilization of the intramolecular fuzzy complex [52]. The interactions of the $\mathrm{SH} 4$ domain with the $\mathrm{SH} 3$ domain or the presence of the Unique domain seems to restrict the extent of oligomerization to dimers or trimers.

Surprisingly, the sequence of the initial segment of the c-Src SH4 domain is found in many snake venom analogues of phospholipase A2, in the juxtamembrane cytoplasmatic side of Adam 10, and, interestingly, in the C-terminal region of K-Ras B isoform, when the sequence is read in reverse order from the single farnesyl modified membrane anchoring element, that plays a similar role of the single myristoylated anchoring element in c-Src (Table 1).

Interestingly, membrane enabled dimerization of Adam 10 was found to depend on the presence of its intrinsically disordered cytoplasmic domain and required the disordered domain to be anchored to the membrane surface [60]. 
Table 1: Putative phospholipid binding sequences in disordered proteins.

\begin{tabular}{|c|c|}
\hline c-Src & myr-GSNKSKPKDASQRRR \\
\hline \multirow{2}{*}{$\begin{array}{l}\text { Snake venoms analogues } \\
\text { of phospholipase } A 2\end{array}$} & NKGKPKDATDR \\
\hline & GRGKPKDATDR \\
\hline Adam 10 cytoplasmic tail & IQQPQRQRPRESYQMGHMRR \\
\hline $\begin{array}{l}\text { K-Ras isoform B } \\
\text { (reverse sequence) }\end{array}$ & far-CKTKSKKKKKKKKG K \\
\hline
\end{tabular}

\section{Outlook: boundaries at different} scales from lipid bilayers to the DBC References

In spite of all their inherent complexity, membrane models provide a conceptual reference for a self-organizing dynamic system built from the assembly of layers with distinct properties, the hydrated layer containing the polar head groups and the hydrophobic core exhibiting a complex phase diagram. The dimensions of the two layers are related by one order of magnitude: roughly $0.5 \mathrm{~nm}$ and $5 \mathrm{~nm}$.

The DBC can be viewed similarly as a self-organizing dynamic system in which the entire lipid membrane is one of the layers while the second layer is formed by the large concentration of disordered proteins tethered to the membrane surface in various ways. In this analogy, the cell membrane would play the role of the head-groups and the disordered proteins would form the core exhibiting collective properties, including protein driven phase transitions and imposing the peculiar environment that we have described as a fluid nanocomposite.

In contrast to the quasi-symmetrical arrangement of the lipid bilayers, the DBC can be better compared with a lipid monolayer facing the cytoskeleton, which is certainly not a rigid barrier. Recent super-resolution microscopy measurements give a first peak of maximum actin density from the cortical cytoskeleton at a distance of around $50 \mathrm{~nm}$ from the cell membrane [61]. The intervening region would correspond to the e-layer highly enriched in disordered proteins but also populated with globular proteins, analogous to the presence of integral membrane proteins in the hydrophobic core of the lipid membrane.

While the properties of individual membrane anchored proteins are obviously important to understand cell signaling and other membrane associated processes, the DBC concept suggests that the collective properties of membrane tethered proteins may be as important to understand these processes, as are the collective properties of lipids to understand the cell membrane.
Acknowledgements: We thank Prof. F. Sagués (Univ. Barcelona), Dr. S. Roche (CNRS, Montpellier), and Dr. A.L Le Roux (IBEC) for insightful discussions. Dr. M. Arbesú calculated the model of the fuzzy complex in Figure 5. This work was supported by a grant from the Spanish government (BIO2016-78006-R) and by the DK in Integrative Structural Biology, funded by the Austrian Science Fund (FWF, project number AW0125821).

Conflict of interest: Authors state no conflict of interest
1. Dyson HJ, Wright PE Intrinsically unstructured proteins and their functions. Nat Rev Mol Cell Biol. 2005;6:197-208.

2. Gsponer J, Futschik ME, Teichmann SA, Babu MM. Tight regulation of unstructured proteins: from transcript synthesis to protein degradation. Science 2008;322:1365-8.

3. Habchi J, Tompa P, Longhi S, Uversky VN. Introducing protein intrinsic disorder. Chem Rev. 2014;114:6561-88.

4. Mittag T, Orlicky S, Choy WY, Tang X, Lin H, Sicheri F, et al. Dynamic equilibrium engagement of a polyvalent ligand with a single-site receptor. Proc Natl Aca. Sci. 2008;105:17772-7.

5. Boeynaems S, Alberti S, Fawzi NL, Mittag T, Polymenidou M, Rousseau F et al. Protein phase separation: a new phase in cell biology. Trends Cell Biol. 2018;28:420-35.

6. Denning DP, Patel SS, Uversky V, Fink AL, Rexach M. Disorder in the nuclear pore complex: The FG repeat regions of nucleoporins are natively unfolded. Proc Natl Acad Sci. 2003;100:2450-5.

7. Lai J, Koh CH, Tjota M, Pieuchot L. Raman V, Chandrababu KB, et al. Intrinsically disordered proteins aggregate at fungal cellto-cell channels and regulate intercellular connectivity. Proc Natl Acad Sci. 2012;109:15781-6.

8. Lingwood D, Simons K. Lipid rafts as a membrane-organizing principle. Science. 2010;327:46-50.

9. lakoucheva LM, Brown CJ, Lawson JD, Obradović Z, Dunker AK. Intrinsic disorder in cell-signaling and cancer-associated proteins. J Mol Biol. 2002;323:573-84.

10. Singer SJ, Nicolson, GL. The fluid mosaic model of the structure of cell membranes. Science 1972;175:720-31.

11. Engelman DM. Membranes are more mosaic than fluid. Nature 2005;438:578-80.

12. Goñi FM. The basic structure and dynamics of cell membranes: An update of the Singer-Nicolson model. Biochim Biophys Acta - Biomembr. 2014;1838:1467-76.

13. Krapf D. Compartimentalization of the plasma membrane. Current Op Cell Biol 2018;53:5-21.

14. Munro S. The golgin coiled-coil proteins of the Golgi apparatus. Cold Spring Harb Perspect Biol 2011;3:a0005256.

15. McEvoy MA, Correll N. Materials that couple sensing, actuation, computation, and communication. Science. 2015;347:1261689.

16. Bürgi J, Xue B, Uversky VN, Van Der Goot FG. Intrinsic disorder in transmembrane proteins: roles in signaling and topology prediction. PLoS ONE, 2016;11:1-21. 
17. Ward JJ, Sodhi JS, McGuffin LJ, Buxton BF, Jones DT. Prediction and functional analysis of native disorder in proteins from the three kingdoms of life. J Mol Biol 2004;337:635-45.

18. Minezaki Y, Homma K, Nishikawa K. Intrinsically disordered regions of human plasma membrane proteins preferentially occur in the cytoplasmic segment. J Mol Biol. 2007;368:90213.

19. Vögler O, Barceló JM, Ribas C, Escribá PV. Membrane interactions of $\mathrm{G}$ proteins and other related proteins. Biochim Biophys Acta - Biomembr. 2008;1778:1640-52.

20. Parsons SJ, Parsons JT. Src family kinases, key regulators of signal transduction. Oncogene 2004;23:7906-9.

21. Wang M, Weiss M, Simonovic M, Haertinger G, Schrimpf SP, et al. PaxDb, a database of protein abundance averages across all three domains of life. Mol Cell Proteomics 2012;11:492-500.

22. Harmon TS, Holehouse AS, Rosen MK, Pappu RV. Intrinsically disordered linkers determine the interplay between phase separation and gelation in multivalent proteins. eLife 2017;6:e30294.

23. Dafforn TR, Smith CJI. Natively unfolded domains in endocytosis: hooks, lines and linkers. EMBO Rep. 2004;5:1046-52.

24. Mouw JK, Ou G, Weaver VM. Extracellular matrix assembly: a multiscale deconstruction. Nat Rev Mol Cell Biol 2014;15:77185.

25. Ponta H, Sherman L, Herrlich P. CD44: from adhesion molecules to signalling regulators. Nat Rev Mol Cell Biol. 2003;4:33-45.

26. Zöller M. CD44: can a cancer-initiating cell profit from an abundantly expressed molecule? Nat Rev Cancer 2011:11:25467.

27. Peeters MC, Van Westen GJP, Li Q, ljzerman AP. Importance of the extracellular loops in $\mathrm{G}$ protein-coupled receptors for ligand recognition and receptor activation. Trends Pharmacol Sci. 2011;32:35-42.

28. Resh MD. Fatty acylation of proteins: new insights into membrane targeting of myristoylated and palmitoylated proteins. Biochim Biophys Acta. 1999;1451:1-16.

29. Bockaert J, Marin P, Dumuis A, Fagni L. The 'magic tail' of G protein-coupled receptors: an anchorage for functional protein networks. FEBS Lett. 2003;546:65-72.

30. El Amri M, Fitzgerald U, Schlosser G. MARCKS and MARCKSlike proteins in development and regeneration. J Biomed Sci. 2018;25:1-12.

31. McLaughlin S, Aderem A. The myristoyl-electrostatic switch: a modulator of reversible protein-membrane interactions. Trends Biochem Sci. 1995;20:272-6.

32. McLaughlin S, Murray D. Plasma membrane phosphoinositide organization by protein electrostatics. Nature 2005;438:605611.

33. Bell Gl. Models for the specific adhesion of cells to cells. Science. 1978;200:618-27.

34. Wu Y, Vendome J, Shapiro L, Ben-Shaul A, Honig B. Transforming binding affinities from 3D to 2D with application to cadherin clustering, Nature, 2011;475:510-3.

35. Bernadó P, García de la Torre J, Pons M. Macromolecular crowding in biological systems: hydrodynamics and NMR methods. J Mol Recognit, 2004;17:397-407.

36. Politou A, Temussi PA. Revisiting a dogma: the effect of volume exclusion in molecular crowding. Curr Opinion Struct Biol. 2015;30:1-6.
37. Busch DJ, Houser JR, Hayden CC, Sherman MB, Lafer EM, Stachowiak JC. Intrinsically disordered proteins drive membrane curvature. Nat Commun. 2015;6:1-11.

38. Lipowsky R. Bending of membranes by anchored polymers. Epl 1995;30:197-202.

39. Zeno WF, Baul U, Snead WT, DeGroot ACM, Wang L, Lafer EM et al. Synergy between intrinsically disordered domains and structured proteins amplifies membrane curvature sensing. Nat Commun. 2018;9:4152.

40. Stachowiak JC, Schmid EM, Ryan CJ, Ann HS, Sasaki DY, Sherman,et al. Membrane bending by protein-protein crowding. Nat Cell Biol. 2012;14:944-9.

41. Truebestein L, Elsner DJ, Fuchs E, Leonard TA. A molecular ruler regulates cytoskeletal remodelling by the Rho kinases. Nat Commun. 2015;6:1-13.

42. Truebestein L, Leonard TA Coiled-coils: the long and short of it. BioEssays 2016;38:903-16.

43. Nagumo H, Sakurada K, Furuya K, Ikuhara T, Hiraoka H, Sasaki $\mathrm{Y}$, et al. Rho-associated kinase phosphorylates MARCKS in human neuronal cells. Biochem Biophys Res Commun. 2001;280:605-9.

44. Teixeira JMC, Fuentes H, Bielskute S, Gairi M, Zėrko S, Koźmiński W, et al. The Two Isoforms of Lyn Display Different Intramolecular Fuzzy Complexes with the SH3 Domain. Molecules. 2018;23:2731.

45. Thomas LL, van der Vegt SA, Fromme JC. A steric gating mechanism dictates substrate specificity of a RAb-GEF. Develop Cell, 2018;48:100-14.

46. Banani SF, Lee HO, Hyman AA, Rosen MK. Biomolecular condensates: organizers of cellular biochemistry. Nat Rev Mol Cell Biol. 2017;18:285-98.

47. Li P, Banjade S, Cheng HC, Kim S, Chen B, Guo L, et al. Phase transitions in the assembly of multivalent signalling proteins. Nature 2012;483:336-40.

48. Banjade S, Rosen MK Phase transitions of multivalent proteins can promote clustering of membrane receptors. Elife. 2014;3:1-24.

49. Su X, Ditlev JA, Hui E, Xing W, Banjade S, Okrut J, et al. Phase separation of signaling molecules promotes $\mathrm{T}$ cell receptor signal transduction. Science. 2016;352:595-9.

50. Bagrodia S, Taylor SJ, Shalloway D. Myristylation is required for Tyr-527 dephosphorylation and activation of pp60_c-src_ in mitosis. Mol Cell Biol 1993;13:1464-70.

51. Pérez Y, Maffei M, Igea A, Amata I, Gairí M, Nebreda AR, et al. Lipid binding by the Unique and SH3 domains of c-Src suggests a new regulatory mechanism. Sci Rep. 2013;3:1295.

52. Arbesú M, Maffei M, Cordeiro TN, Teixeira JMC, Pérez Y, Bernadó $P$, et al. The Unique Domain Forms a Fuzzy Intramolecular Complex in Src Family Kinases. Structure 2017; 25: 630-40.

53. Le Roux AL, Mohammad I-L, Mateos B, Arbesú M, Khan FA, Teixeira JMC, et al. A Myristoyl Binding Site in the SH3 Domain Modulates c-Src Membrane Anchoring iScience 2019;12:194203.

54. Spassov DS, Ruiz-Saenz A, Piple A, Moasser MM. A dimerization function in the intrinsically disordered $\mathrm{N}$-terminal region of src. Cell Rep. 2018;25:449-63.

55. Le Roux AL, Busquets MA, Sagués F, Pons M. Kinetics characterization of c-Src binding to lipid membranes: Switching from labile to persistent binding. Colloids Surfaces B Biointerfaces 2016;138:17-25. 
56. Le Roux AL, Castro B, Garbacik ET, Garcia Parajo MF, Pons $M$. Single molecule fluorescence reveals dimerization of myristoylated Src N-terminal region on supported lipid bilayers. ChemistrySelect 2016;1:642-47.

57. Owen DM, Rentero C, Rossy J, Magenau A, Williamson D, Rodriguez M, et al. PALM imaging and cluster analysis of protein heterogeneity at the cell surface. J Biophotonics 2010;3:446-54.

58. Smith AW, Huang HH, Endres NF, Rhodes C, Groves JT. Dynamic organization of myristoylated src in the live cell plasma membrane. J Phys Chem B 2016;120:867-76.

59. Dwivedi M, Mejuch T, Waldmann H, Winter R. Lateral organization of host heterogeneous raft-like membranes altered by the myristoyl modification of tyrosine kinase c-src. Angew Chemie - Int Ed. 2017;56:10511-5.

60. Deng W, Cho S, Su P-C, Berger BW, Li R. Membrane-enabled dimerization of the intrinsically disordered cytoplasmic domain of ADAM10. Proc Natl Acad Sci USA 2014;111:15987-92.

61. Clausen MP, Colin-York H, Schneider F, Eggeling C, Fritzsche $M$ Dissecting the actin cortex density and membrane-cortex distance in living cells by super-resolution microscopy. J Phys D: Appl Phys 2017;50:064002. 